\title{
HPSTOS: High-Performance and Scalable Traffic Optimization Strategy for Mixed Flows in Data Center Networks
}

\author{
Yong Liu, Huaxi Gu, and Ning Wang
}

\begin{abstract}
In data center networks, traffic needs to be distributed among different paths using traffic optimization strategies for mixed flows. Most of the existing strategies consider either distributed or centralized mechanisms to optimize the latency of mice flows or the throughput of elephant flows. However, low network performance and scalability issues are intrinsic limitations of both strategies. In addition, the current elephant flow detection methods are inefficient. In this paper, we propose a high-performance and scalable traffic optimization strategy (HPSTOS) based on a hybrid approach that leverages the advantages of both centralized and distributed mechanisms. HPSTOS improves the efficiency of elephant flow detection through sampling and flow-table identification. HPSTOS guarantees preferential transmission of mice flows using priority scheduling and adjusts their transmission rate by coding-based congestion control on the end-host, reducing their latency. Additionally, HPSTOS schedules elephant flows by cost-aware dynamic flow scheduling on a centralized controller to improve their throughput. The controller handles only elephant flows, which constitutes the minority of the flows, allowing effective scalability. Evaluations show that HPSTOS outperforms existing schemes by realizing efficient elephant flow detection and improving network performance and scalability.
\end{abstract}

Index Terms-Data center networks, Traffic optimization, High performance, Scalability.

\section{INTRODUCTION}

W ITH the expansion of network scale and the growth in burst traffic [1-5], data center networks (DCNs) require a high-performance and scalable traffic optimization strategy to distribute traffic among the shortest paths. Data center traffic optimization strategies include flow scheduling, congestion control, load balancing, and routing methods [6]. The mixed flows in the data center network include two categories of data flows with different features: elephant flows and mice flows [7]. Elephant flows require a long transmission time owing to their large volumes, and they require higher throughput. In contrast, mice flows typically require a short transmission time as they are usually latencysensitive [5]. Normally, the elephant and mice flows travel on the same path. However, frequent resource competition and conflicts are inevitable when elephant and mice flows share transmission paths [8]. For example, mice flows that are blocked by elephant flows result in long flow completion times (FCT). When multiple elephant flows with large bandwidth requirements share the transmission path, hash collisions occur, resulting in a lower throughput for elephant flows.

To improve network performance, existing traffic optimization strategies are mainly divided into two categories: optimization strategies to solve the blocking problem of mice flows and optimization strategies to solve the hash collision problem of elephant flows. Optimization strategies

- Y. Liu and H.X. Gu are with the State Key Laboratory of Integrated Service Networks (ISN), Xidian University, Xi'an 710071, China.

E-mail: yong.liu.xidian@foxmail.com, hxgu@xidian.edu.cn.

- N. Wang is with the Institute for Communication Systems (ICS), University of Surrey, Guildford, Surrey GU2 7XH, U.K.

E-mail: n.wang@surrey.ac.uk. for mice flows are usually deployed on the end-host by a distributed mechanism and use a congestion control method to reduce the latency of the mice flows. For example, the typical congestion control schemes DCTCP [9] and $\mathrm{D}^{2} \mathrm{TCP}$ [10] adaptively adjust the traffic transmission rate according to the congestion state of the network. When the buffer queue of the switch is crowded, as indicated by congestion feedback, the sending rate of the traffic is reduced to alleviate network congestion. Although these congestion control schemes can optimize the latency of mice flows, the throughput demand of elephant flows are not adequately addressed. Additionally, in a large-scale network, the endhost needs to store many congestion feedback messages, resulting in a large storage overhead $[9,10]$.

Optimization strategies for elephant flows usually apply a centralized mechanism deployed on a centralized controller that improve the throughput of elephant flows through flow scheduling. For example, the typical elephant flow scheduling solutions, Hedera [11] and Freeway [12], collect the status information of all paths using the controller and schedule the elephant flows to a path with a lighter load. However, most existing elephant flow scheduling schemes perceive link utilization as the link status without considering the marginal cost of the link, which can reduce the overall throughput of the network. Further, they cannot achieve a good trade-off between the latency of mice flows and the throughput of elephant flows. Additionally, in a large-scale network, the controller needs to process many flow request messages, resulting in lower scalability owing to the capacity limitation of the controller.

Further, the current elephant flow detection methods are mainly divided into three categories: controller-based detection, end-host-based detection, and switch-based de- 
tection. The controller-based detection method identifies an elephant flow by collecting all flow request messages and calculating the size of the flow, which causes considerable controller overhead. To reduce this overhead, the end-hostbased detection method detects the elephant flow by setting a threshold in the socket buffer of the host. However, in practice, it is difficult to implement as it needs to modify the protocol stack of the host. Switch-based detection identifies elephant flows by sampling the flow, which greatly reduces the detection overhead; however, its detection accuracy is low.

In this study, we designed and evaluated a highperformance and scalable traffic optimization strategy $(\mathrm{H}-$ PSTOS) for mixed flows. To distinguish between elephant flows and mice flows, HPSTOS uses a low-cost and highaccuracy elephant flow detection method. To meet the performance requirements of the elephant and mice flows concurrently, HPSTOS jointly uses distributed and centralized mechanisms. First, to meet the low latency requirements of mice flows, HPSTOS adopts a distributed mechanism, deploys a coding-based congestion control method on the end-host, and sets priority scheduling on the switch for priority transmission of mice flows. Second, to meet the high throughput requirements of elephant flows, HPSTOS uses a centralized mechanism and deploys a cost-aware flow scheduling method on a centralized controller. Because the controller will only process the request messages of elephant flows and elephant flows only account for approximately $10 \%$ of all traffic, HPSTOS is scalable.

This paper comprehensively addresses fairness issues between elephant and mice flows and individual performance optimality, which has not yet been adequately addressed in existing literature. The specific contributions of this study are as follows:

- Unlike traditional elephant flow detection, HPSTOS used an efficient elephant flow detection to reduce detection overhead and improve detection accuracy. First, the flow was sampled in order to detect suspected elephant flows, reducing the controller overhead. Then, the controller generated a flow table entry for the suspected elephant flow to recognize it, thereby improving detection accuracy. Performance evaluation showed that when compared with existing detection methods, HPSTOS had better detection performance in terms of overhead and accuracy.

- To improve the overall performance of the network, unlike existing traffic optimization strategies, HPSTOS used both distributed and centralized mechanisms. HPSTOS used priority scheduling for priority transmission of mice flows and adaptively adjusted the transmission rate of the mice flows through coding-based congestion control. At the same time, when the congestion was severe it could reduce the retransmission delay, caused by packet loss, by decoding redundant packets. In addition, HPSTOS used cost-aware elephant flow scheduling to dynamically schedule elephant flows to lighter-load paths by sensing the marginal cost of the link. The simulation showed that compared with existing traffic optimization strategies, HPSTOS could reduce the FCT of mice flows by approximately $40 \%$ and increase the throughput of elephant flows by approximately $35 \%$.

- To allow for scalability, in contrast to existing traffic optimization strategies, HPSTOS used a distributed mechanism to handle mice flows and a centralized mechanism to handle elephant flows. The HPSTOS processed request messages of the elephant flows through the controller and the congestion feedback information of mice flows through the end-host. The simulation showed that HPSTOS had better scalability than the existing solutions.

The remainder of this paper is organized as follows. We describe related work in Section 2. Section 3 presents the design decisions of HPSTOS and Section 4 describes the detailed design of HPSTOS. In Section 5, the performance is evaluated using the Mininet simulation. Finally, the paper is concluded in Section 6.

\section{Related Work}

For the transmission of mixed flows among multiple paths, elephant flow detection and traffic optimization strategies for DCNs have been studied. The related works are discussed next.

\subsection{Elephant Flow Detection}

For elephant flow detection, the existing research work is mainly divided into three categories including controllerbased detection, host-based detection, and switch-based detection methods. A detailed introduction and analysis of the various schemes are discussed.

Controller-based detection methods detect an elephant flow by statically monitoring the forwarding rate of flows. Hedera [11] recognizes flows as elephant flows if their forwarding rates exceed $100 \mathrm{Mbps}$. Helios [13] extracts the per-flow rates from each of its edge-switches using a centralized controller. Whenever the flow rate exceeds the threshold, an elephant flow is detected. Flight [14] is a fast and lightweight elephant flow detection method that leverages transmission control protocol (TCP) communication behavior. To measure the flow rate, the above methods periodically poll flow entries from switches and calculate the flow rates at the controller. Due to bursty traffic in DCNs, long polling periods result in inadequate detection performance and shorter polling periods lead to a higher controller overhead. For example, in a data center with 500 edge-switches, with 120 flow entries per edge-switch [15], and a polling period of $0.01 \mathrm{~s}$, the controller must collect 6000000 entries per second, generating about $500 \mathrm{Mbps}$ of traffic. Therefore, the controller-based detection method results in a higher controller overhead through poll-based information collection.

Host-based detection methods allocate elephant flow detection to the operating systems of hosts to avoid the controller overhead caused by the controller-based detection methods. Mahout [16] proposes the detection of elephant flows in the socket buffer of the end-host. When the flow size exceeds a threshold, e.g., $100 \mathrm{~KB}$, the flow is measured as an elephant flow. However, this method requires the modification of the protocol stack of the host for continuous operation, which 
is complicated and challenging to achieve in an actual DCN [17].

Switch-based detection methods detect elephant flows by using a sampling method, achieving good balance between detection overhead and implementation complexity. NetFlow [18] and sFlow [19] provide a fine-grained detection method by sampling packets. For packet sampling, the switch captures one of every $N$ packets on each input port. For a specified period, the sampling activity is performed twice and the header information of the sampled packets is sent to the controller. The controller then parses the fivetuple of the packets. If two packets are from the same flow, the flow is marked as an elephant flow. Mori et al. [20] developed schemes to determine the threshold of sampled packets for a single flow based on Bayes' theorem. However, frequent detection errors when using sampling, results in low elephant flow detection accuracy. Because sampling detection generally uses random sampling or probabilistic sampling, it may result in mice flows to be mistaken for elephant flows.

\subsection{Traffic Optimization for Mixed Flows in Data Center Networks}

To effectively distribute traffic among multiple paths, many traffic optimization strategies for mixed flows have been proposed in recent years. These schemes are mainly classified into optimization strategies for mice flows and optimization strategies for elephant flows. The details are as follows.

\subsubsection{Optimization strategies for mice flows}

When mice and elephant flows share transmission paths, mice flows might potentially be blocked by elephant flows, resulting in long FCT. To reduce the FCT of mice flows and meet their low latency requirements, existing optimization strategies for mice flows are mainly divided into host-based rate control and switch-based priority scheduling schemes. Based on the end-host, DCTCP [9] uses explicit congestion notification (ECN)-based adaptive congestion control and appropriate throttling of elephant flows to keep the switch queue occupancy low, reducing mice flows' FCT. DCTCP can adaptively adjust the congestion window of flows according to the level of link congestion. However, DCTCP does not consider the urgency of the flows, e.g., deadlines, which results in the ineffective reduction of the FCT of mice flows. $\mathrm{D}^{2} \mathrm{TCP}$ [10], $\mathrm{D}^{3}$ [21], and PDQ [22] use explicit deadline information to drive rate allocation, congestion control, and preemptive scheduling decisions. Although the above schemes can reduce the FCT of mice flows, severe congestion can cause packet loss on the switch buffer queue bringing about packet retransmission; consequently, lengthening the latency of mice flows.

Additionally, using the switch, RepFlow [23] copies the mice flows and forwards them to different paths to achieve a lower FCT. A multi-level feedback queue (MLFQ) [24] can efficiently solve flow scheduling without size information, as shown by PIAS [25], when the only objective is to minimize the FCT. In a mixed-flow setting where both deadline miss rate and FCT need to be considered, it is not clear how deadline and non-deadline flows should be prioritized against each other to achieve the best performance. In addition, PIAS cannot distinguish between elephant and mice flows, which could cause an elephant flow to be scheduled to the high-priority queue. Karuna [26] schedules deadline flows to high-priority queues and handles them through a distributed rate control protocol. Non-deadline flows are placed in the lower priority queues and uses an extended PIAS scheduling algorithm to jointly schedule non-deadline flows with known or unknown sizes. AuTO [6] uses MLFQ to schedule long and short flows and deep reinforcement learning to optimize the threshold of priority queues, which reduces the latency of short flows. pFabric [27] achieves superior performance by approximating the ideal shortest job first through decentralized in-network prioritization. However, it assumes complete knowledge of the flow sizes. Therefore, for actual data traffic from the DCN, pFabric cannot identify the flow size and distinguish between elephant and mice flows.

Although the above schemes can effectively reduce the latency of mice flows, the throughput demand of elephant flows cannot be adequately satisfied. The improvement in latency of mice flows is at the expense of throughput of elephant flows.

\subsubsection{Optimization strategies for elephant flows}

When multiple elephant flows share the same transmission path, large bandwidth requirements may cause hash collisions. In order to effectively schedule elephant flows and improve their throughput, existing optimization strategies for elephant flows mainly include centralized and distributed load balancing. For centralized load-balancing schemes, Hedera [11] uses OpenFlow technology to dynamically estimate the required bandwidth of elephant flows and then schedules them to the path with the lowest link utilization, improving throughput. SDN-LB [28] dynamically monitors the load status of the network and can adaptively reroute traffic from a heavier-load path to a lighter-load path, which improves the throughput of elephant flows. FastPass [29] is an ideal centralized approach. It not only controls the flow's forwarding paths but also determines the time at which each packet should be transmitted. However, to accurately schedule every packet in the network, very stringent time synchronization is required to achieve submicrosecond synchronization. Thus, the system is highly precise and sophisticated, which limits its scalability. Freeway [12] equally partitions all paths into low-latency paths and high-throughput paths for mice flows and elephant flows, respectively. Partitioning paths avoid competition between the two types of flows. DiffFlow [30] is a scheduling scheme that distinguishes between the mixed flows and uses random packet scatter [31] technology and hash matching to forward elephant and mice flows, respectively. The above centralized load-balancing schemes effectively improves the throughput of the elephant flow; however, the latency performance of mice flows is not sufficiently addressed. In addition, the status information of the entire network is collected by a centralized controller. Due to the capacity limitation of the controller, scalability is affected, and therefore, these solutions are not suitable for a largescale network. 
For distributed load-balancing schemes, FLARE [32] can systematically split a single flow across multiple paths without causing packet reordering. In this process, the flow is split into flowlets of different sizes through the burstiness of the flow. LocalFlow [33] is a switch-local algorithm implemented in OpenFlow-enabled switches. By splitting individual flows spatially and assigning them to proper paths, it performs well in symmetric topologies. MPTCP [34] leverages multiple paths between end-hosts and sets up multiple sub-connections to make full use of the bandwidth and improve throughput. CONGA [35] obtains global congestion information by sending probe packets and routes the flowlet granularity to the path with the least congestion. Presto [36] divides the traffic into flowcells and uses round-robin or weighted scheduling to forward the flowcells to different paths. LetFlow [37] can flexibly adjust the size of the flowlet by controlling the sending rate of the flow to adapt to the congestion state of different paths. DRILL [38] selects the output ports of the switch to control congestion. When a packet arrives at the switch, DRILL selects an output port with the least congestion. Hermes [39] perceives path congestion through ECN and RTT and uses a heuristic method to reroute packets to the leastcongested, fault-free paths. HULA [40] routes the flowlet to the best next hop using hop-by-hop congestion monitoring. The above distributed solutions have superior performance in solving the hash collision problem and achieve higher throughput of elephant flows. However, they cannot achieve a good balance between the latency of mice flows and the throughput of elephant flows.

\section{HPStOS Design Strategies}

In this section, we will discuss the three key aspects influencing the decision to use joint mechanisms to optimize mixed flows. Key design decisions in HPSTOS included distinguishing between elephant and mice flows and jointly using distributed and centralized schemes.

\subsection{Necessity of Distinguishing Between Elephant Flows and Mice Flows}

\subsubsection{Need for flow-level granularity}

In the DCN, the granularity of transmission between multipaths mainly comprises the flow level, flowlet level, flowcell level, and packet level. It is generally acknowledged that packets with the same five-tuple information (source and destination IP, source and destination port, and protocol) form a flow. During the load-balancing activity, only the first packet of the flow is hashed to calculate the flow forwarding table. When the other packets of the flow arrive at the switch the forwarding table is queried to complete the forwarding function. Therefore, load balancing based on flow granularity reduces the overhead by hash matching to the first packet of the flow and avoids the out-of-order problem caused by packet transmission.

In accordance with TCP, packets are sent in bursts, which causes a time delay between data packets reaching the switch. Following the characteristics of TCP, flowlet technology [32] cuts the flow into multiple flow slices, called flowlets. A new flowlet is generated when the time interval

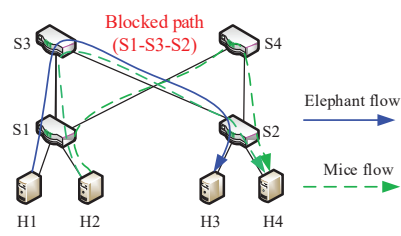

(a)

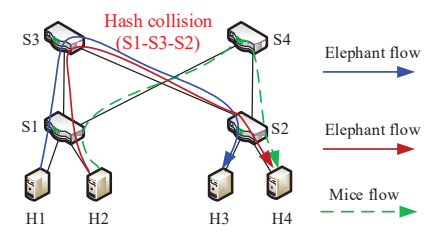

(b)
Fig. 1. An example that resource competition between mixed flows.

between the arrival of two packets exceeds a threshold. The flowcell granularity is a fixed $64 \mathrm{~KB}$ unit. The size of the TCP Segment Offload in existing virtual machines is $64 \mathrm{~KB}$. Therefore, the resulting size of the flowcell in Presto [36] is $64 \mathrm{~KB}$, reducing the possibility of the flow being divided during multipath forwarding. The packet granularity is the smallest transmission unit. At the switch, each packet is polled for routing decisions through hash operations. Although the aforementioned fine-grained transmission units, flowlet, flowcell, and packet, can improve the transmission efficiency of traffic between multiple paths, fine-grained load balancing requires calculations for many forwarding rules, which increases overhead and causes the out-of-order packet problem.

Therefore, we used flow-level transmission units in HPSTOS and divided the mixed flows in the DCN into elephant flows and mice flows. Our flow-level design reduced the large overhead for calculating forwarding rules and avoided the out-of-order problem of data packets.

\subsubsection{Need of solving resource competition between mixed flows}

In an actual DCN, resource competition and conflicts are common when elephant and mice flows share transmission paths. For example, mice flows could potentially be blocked by elephant flows, resulting in a longer FCT. In addition, hash collisions may occur between multiple elephant flows on the same transmission path owing to their high bandwidth requirements.

An example of a mice flow blocked by elephant flow is shown in Fig. 1(a). Server H2 sends a mice flow to H4, half of the flow is routed through path S1-S3-S2, and the other half flows through path S1-S4-S2. The server H1 sends the elephant flow to H3, using the path S1-S3-S2. Because the network cannot distinguish between the elephant flow and the mice flow, the mice flow is blocked by the elephant flow on path S1-S3-S2, as the latter consumes a large amount of long-lived network bandwidth. The hash collisions between multiple elephant flows are shown in Fig. 1(b). The elephant flow from server $\mathrm{H} 1$ to $\mathrm{H} 3$ and the elephant flow from $\mathrm{H} 2$ to H4 share a transmission path S1-S3-S2, causing a hash collision between the two elephant flows. As the network cannot recognize the elephant flows, the path S1-S3-S2 is overloaded.

In HPSTOS, we needed to distinguish between elephant flows and mice flows to solve the resource competition between mixed flows. Existing elephant flow detection schemes have a large detection overhead and low detection accuracy. Therefore, we considered the design of a low-cost and high-accuracy elephant flow detection method. 


\subsection{Need for High Performance}

For the mixed flow in the DCN, the elephant flow requires high throughput, and the mice flow is sensitive to latency. To solve the problem of mice flows being blocked by elephant flows, optimization strategies for mice flows are proposed to reduce the latency of the mice flow. Existing strategies are usually deployed on end-hosts or switches through a distributed mechanism for rate control or priority scheduling. Although most solutions can effectively reduce the latency of mice flows, some challenges still exist. For example, the existing rate control schemes DCTCP [9] and $\mathrm{D}^{2} \mathrm{TCP}$ [10] will cause packet loss and retransmission when the queue is heavily congested, causing long delays. Moreover, the existing priority-scheduling schemes pFabric [27] and PIAS [25] cannot distinguish between elephant flows and mice flows, which may cause the elephant flow to be scheduled to a high-priority queue. Therefore, mice flow latency issues are not adequately addressed. In addition, schemes that effectively optimize the latency of mice flows, do not always adequately optimize the throughput of elephant flows.

To solve the problem of hash collisions between elephant flows, optimization strategies for elephant flows are proposed to improve the throughput of elephant flows. They are usually deployed on a centralized controller for dynamic scheduling of traffic through a centralized mechanism. They collect the state of the entire network through the controller and schedule the elephant flow to the lightest-load path. Although the throughput of the elephant flow can be effectively improved by sensing the path status, there are still some challenges. For example, Hedera [11] and Freeway [12] only perceive the link utilization rate but not the marginal cost of the link, which leads to lower energy efficiency. Additionally, these solutions cannot achieve a good tradeoff between the latency of mice flows and the throughput of elephant flows.

In summary, we can see that most existing solutions cannot achieve a good balance between the throughput of elephant flows and the latency of mice flows. In HPSTOS, we jointly used distributed and centralized mechanisms to improve network performance. The design strategies of HPSTOS were as follows: (1) To reduce the latency of mice flows, we proposed a distributed mechanism. In designing priority scheduling, we determined the type of flow at the switch and gave preference to urgent mice flows. The mice flows and elephant flows were scheduled to the highpriority and low-priority queues, respectively. In addition, we used coding-based congestion control at the end-host to reduce the congestion state of mice flows and avoid the delay caused by packet retransmission. (2) To improve the throughput of the elephant flow, we proposed the use of a centralized mechanism. On the controller, we deployed a cost-aware dynamic flow scheduling method to sense the marginal cost of different paths, schedule the elephant flow to the lightest-load path, and improve the throughput of elephant flows. Through this hybrid mechanism, a good trade-off can be achieved between the throughput of elephant flows and the latency of mice flows.

\subsection{Need for High Scalability}

In the DCN, the mixed flow is composed of elephant flows and mice flows. The mice flows account for more than $85 \%$ of the total traffic; however, it only accounts for approximately $15 \%$ of the total capacity. In contrast, elephant flows account for more than $85 \%$ of the total capacity, while the quantity is relatively small at approximately $15 \%$ [41].

The existing distributed congestion control schemes DCTCP [9], D 2 TCP [10], and PDQ [22] are deployed on the end-host. ECN marking information of traffic is used to feed back the congestion status of the path. As the network scale expands, the end-host needs to store larger amounts of ECN marking information resulting in a large storage overhead. RepFlow [23] is deployed on the switch and copies the mice flow to all equivalent paths. In a large-scale network, there are many equal-cost multipaths between the source and destination switch pairs. Therefore, RepFlow will result in greater network energy consumption and lower scalability. In addition, distributed load-balancing solutions, CONGA [35] and HULA [40], are deployed on the switch. They store a large amount of congestion information on the switch obtained through path congestion feedback. As a network expands, the scalability of this solution is low and dependent on the limitation of the storage capacity of the switch.

The existing centralized load-balancing solutions Hedera [11], Freeway [12], and DiffFlow [30] are deployed on a centralized controller. They make routing decisions for traffic based on network status information. When a flow reaches the switch, it is matched to the flow table on the switch. If there is no corresponding flow table entry, a packet_in request message is sent to the centralized controller. After the controller receives the request message, it collects all path status information and calculates the flow entries. Therefore, in a large-scale network, the controller will need to process a large number of request messages. Therefore, the capacity of the controller limits this solution and the scalability thereof.

Based on the analysis above, we derived the design strategies of HPSTOS as follows: (1) In HPSTOS, we jointly used distributed and centralized mechanisms. (2) A centralized controller was responsible for scheduling the elephant flows, allowing for scalability as only a small number of elephant flow request messages would be generated and processed. (3) The mice flows were processed through a distributed mechanism at the end-host, using an ECN to mark the mice flows and feed back its congestion status. This allowed HPSTOS to reduce the overhead of storing congestion information on larger scale networks.

\section{Detalled Design of HPStos}

According to the design strategies described in Section 3, we used both distributed and centralized mechanisms to design the HPSTOS system framework. Fig. 2 shows the HPSTOS systems components. On the switches, the elephant flow detection component was responsible for distinguishing between elephant and mice flows. The function of this component was realized by an effective switch selection algorithm and an elephant flow detection algorithm. The effective switch selection algorithm first selected switches used for sampling, and then passed the selected switch 


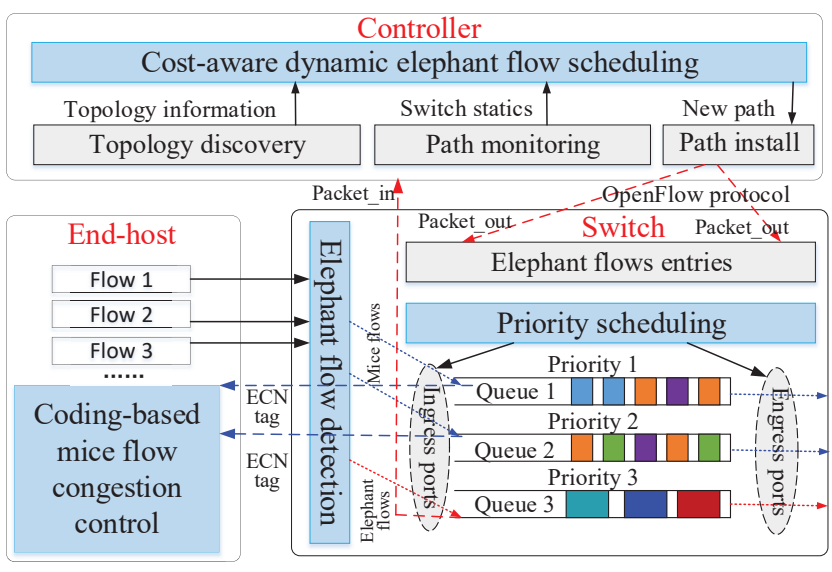

Fig. 2. System structure of the HPSTOS.

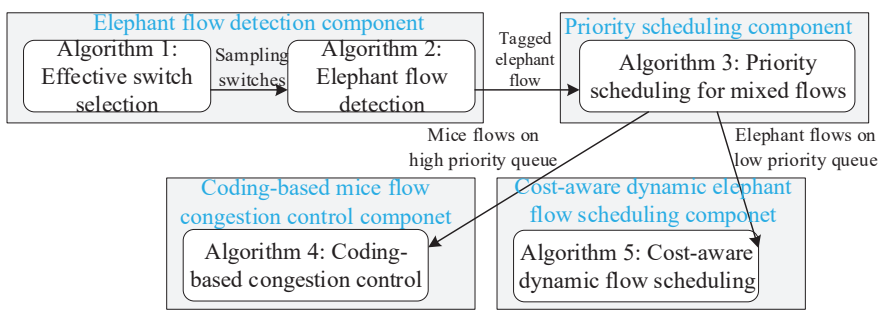

Fig. 3. Connection relationship between algorithms.

to the elephant flow detection algorithm for elephant flow detection. In addition, the priority scheduling component on the switches scheduled the mice flows and the elephant flows to the high-priority and low-priority queues, respectively. The function of this component was realized using a priority scheduling algorithm. To deal with mice flows, HPSTOS used a distributed mechanism. On the end-hosts, the coding-based mice flow congestion control component adaptively adjusted the transmission rate of mice flows through an algorithm to reduce their congestion status. Simultaneously, when the buffer queue was heavily congested, the algorithm used forward error correction (FEC) technology to reduce the retransmission delay caused by packet loss. Further, to deal with elephant flows, HPSTOS used a centralized mechanism. In the controller, the cost-aware dynamic elephant flow scheduling component monitored all path state information and dynamically scheduled elephant flows to the lightest-load path through an algorithm to improve their throughput. In the HPSTOS system, we proposed five different algorithms. The relationship between these algorithms is shown in Fig. 3.

In summary, HPSTOS distinguished between mice and elephant flows using elephant flow detection, and scheduled mice flows to high-priority queues on the switch for priority transmission using priority scheduling. In high-priority queues, when mice flows were congested, the packets of the mice flows were tagged with the ECN and the congestion information was passed to the source server. Based on the degree of congestion, the source server adaptively adjusted its transmission rate through coding-based mice flow congestion control, reducing latency. In addition, the elephant flows were scheduled to a low-priority queue via priority scheduling. The packet_in message was sent to the controller only when the routing table did not have a flow entry for the incoming elephant flow. According to the status information of all paths, the controller made routing decisions through cost-aware dynamic elephant flow scheduling and sent them to the switch in the form of a packet_out message. Therefore, the elephant flows could be scheduled to the lightest-load path, improving their throughput. In Section 4.5, we will provide more details on the interaction and dependency between each component.

\subsection{Elephant Flow Detection}

To effectively detect the elephant flow, we designed a lowcost and high-accuracy elephant flow detection method. Unlike traditional sampling methods, we first detected the suspected elephant flows by sampling, and then accurately identified the elephant flows by querying the flow table. We reduced the resource overhead of the controller by using sampling and a flow table query was used to improve the accuracy of elephant flow detection.

Owing to the constraints of the routing strategy, different flows may pass through different switches. Assuming that the network was interconnected by three switches and generated a total of three flows, namely $f_{1}, f_{2}$, and $f_{3}$. According to routing constraints, the flow sets passing through switches $s_{1}, s_{2}$, and $s_{3}$ were $\left\{f_{1}, f_{3}\right\},\left\{f_{2}, f_{3}\right\}$, and $\left\{f_{2}\right\}$, respectively. Given that packets were sampled at each switch, flow $f_{1}$ was sampled once, and flows $f_{2}$, and $f_{3}$ were each sampled twice. In fact, if only the switches $s_{1}$ and $s_{3}$ were sampled, every flow would have been sampled exactly once, reducing the resource overhead compared to sampling on each switch.

Therefore, in the process of flow sampling, determining the appropriate switches for sampling is a problem that must be solved. Given $j$ switches in the network that could be used for sampling, denoted as $V=\left\{s_{1}, s_{2}, \ldots, s_{j}\right\}$, and $i$ flows, denoted by $F=\left\{f_{1}, f_{2}, \ldots, f_{i}\right\}$. A matrix $\Omega_{i \times j}$ was constructed to indicate whether $f_{i}$ passed through switch $s_{j}$.

$$
\Omega_{i \times j}=\left\{\begin{array}{cccccc}
\mu_{1,1} & \mu_{1,2} & \mu_{1,3} & \cdots & \mu_{1, b-1} & \mu_{1, j} \\
\mu_{2,1} & \mu_{2,2} & \mu_{2,3} & \cdots & \mu_{2, b-1} & \mu_{2, j} \\
\vdots & \vdots & \vdots & \vdots & \vdots & \vdots \\
\mu_{i, 1} & \mu_{i, 2} & \mu_{i, 3} & \cdots & \mu_{i, j-1} & \mu_{i, j}
\end{array}\right\}
$$

For any element $\mu_{i, j}$ in $\Omega_{i \times j}$, the value satisfies the constraint in Equation (2). If the flow $f_{i}$ passes through the switch $s_{j}$, the value of $\mu_{i, j}$ is 1 ; otherwise it is 0 .

$$
\mu_{i, j}= \begin{cases}1, & f_{i} \rightarrow s_{j}, \\ 0, & \text { otherwise } .\end{cases}
$$

While ensuring that all traffic was sampled, our goal was to choose as few switches as possible for flow sampling. As shown in Algorithm 1, we proposed an effective switchselection algorithm for generating a set of sampling points. First, for the relationship between the flows and switches, a matrix $\Omega_{i \times j}$ was constructed. Based on the values of all elements in the matrix $\Omega_{i \times j}$, the flow set $F\left(s_{j}\right)$ passing through 
each switch $s_{j}$ could be calculated. Then, the switches $s_{j}$ were sorted according to the size of $F\left(s_{j}\right)$, and the switch set $V^{*}$ to be selected was generated. Finally, all switches $s_{j}$ in the set $V^{*}$ were traversed sequentially. Under the constraint that all traffic was to be sampled, the appropriate switches $s_{j}$ were selected as the sampling points and added to the sampling point set $\Theta$.

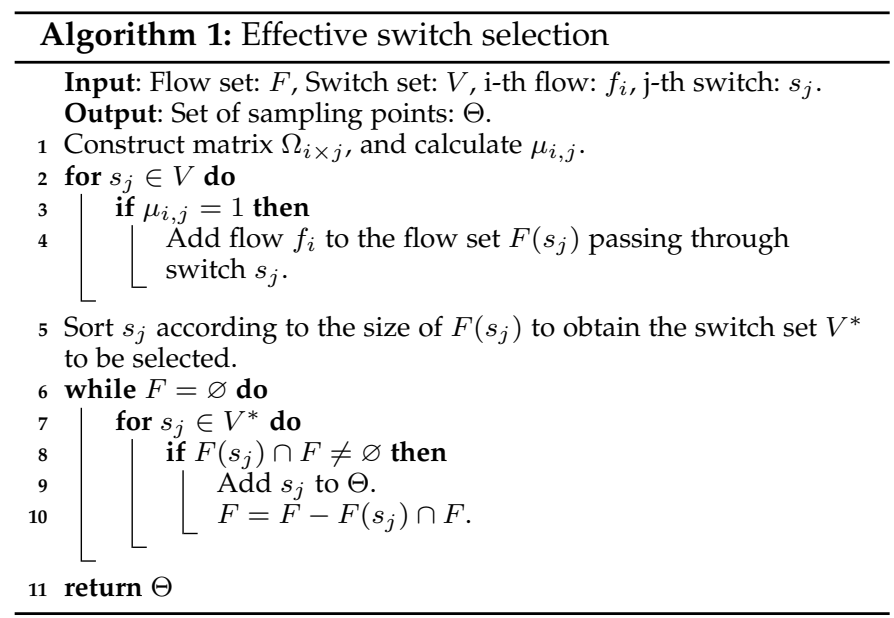

After determining the sampling points, each switch sampled packets according to the sampling rate $\varepsilon$ set in advance and maintained a hash table $T_{-}$hash for the sampled flow $f_{i}$ through the collector sFlow [42]. When packet $p$ was sampled, $p$ was judged to belong to $f_{i}$ if $f_{i}=\operatorname{Hash}\left(i d_{p}\right)$. If $p$ belonged to flow $f_{i}$, collector sFlow increased the value of $T \_h a s h\left(f_{i}\right)$ by volume $(p)$. Once the value of $T \_h a s h\left(f_{i}\right)$ exceeded the set threshold $\rho$, the flow $f_{i}$ was marked as a suspected elephant flow, and collector sFlow immediately sent the request message of $f_{i}$ to the controller.

After receiving the request message of flow $f_{i}$, the controller calculated the flow table entry and sent it to the switch to measure the actual elephant flow. Then, the controller read the flow table entry to obtain the actual data volume of flow $f_{i}$. If the data volume of the acquired flow $f_{i}$ was greater than $\sigma, f_{i}$ was identified as an actual elephant flow. The specific elephant flow detection process is presented in Algorithm 2.

\subsection{Priority Scheduling}

In a DCN, existing priority-scheduling schemes pFabric [27] and PIAS [25] schedule traffic by setting multiple priority queues in the switch buffer. Although the FCT of the flow can be effectively reduced by priority scheduling, flow sizes are not monitored, which can lead to elephant flows being scheduled to the high-priority queue. If the elephant flow is transmitted through a high-priority queue, mice flows will be blocked, resulting in longer FCT for the mice flows. In [43], a deadline-aware queue scheme is proposed. It establishes three priority queues and schedules flows by sensing the deadline. However, it does not consider the shortest remaining processing time of the flow.

To avoid blocking the mice flows, we proposed a priority-scheduling algorithm to assign queues for mixed flows. HPSTOS provided three priority queues available in each port buffer, descending in order of priority. Packets

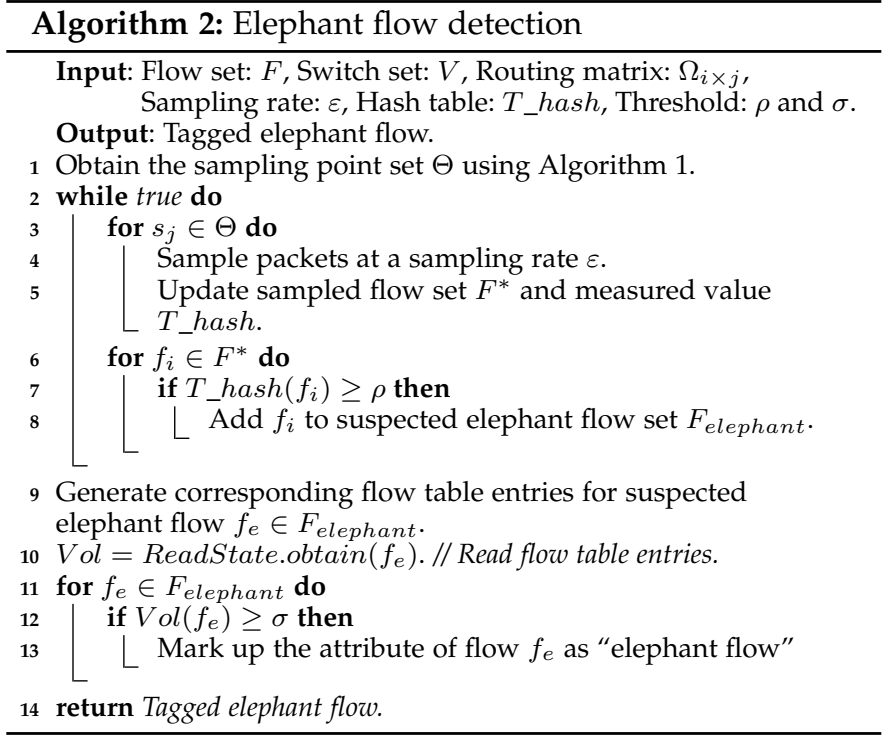

in different queues were scheduled with strict priority, whereas packets in the same queue were scheduled based on the first in, first out principle. When the high-priority queue was empty, the packets of the low-priority queue were scheduled. When assigning priority to the mixed flow, elephant flows were scheduled to the low-priority queue, and mice flows were scheduled to the high-priority queue.

Simultaneously, we considered the urgency of mice flows; mice flows with high urgency were given higher priority. Equation (3) defines the urgency $u$ of the mice flow, where $T_{\lambda}$ is the running time and $T_{\varphi}$ is the deadline for this flow. We set a time stamp function in the IP packet header to enable the calculation of the running time, $T_{\lambda}$, of the flow. All OVS ports were monitored through a softwaredefined network (SDN) controller to obtain the time stamp. The urgency $u$ of the flow increased as the deadline neared, creating the need for more bandwidth to expedite transmission.

$$
u=\frac{T_{\lambda}}{T_{\varphi}-T_{\lambda}} .
$$

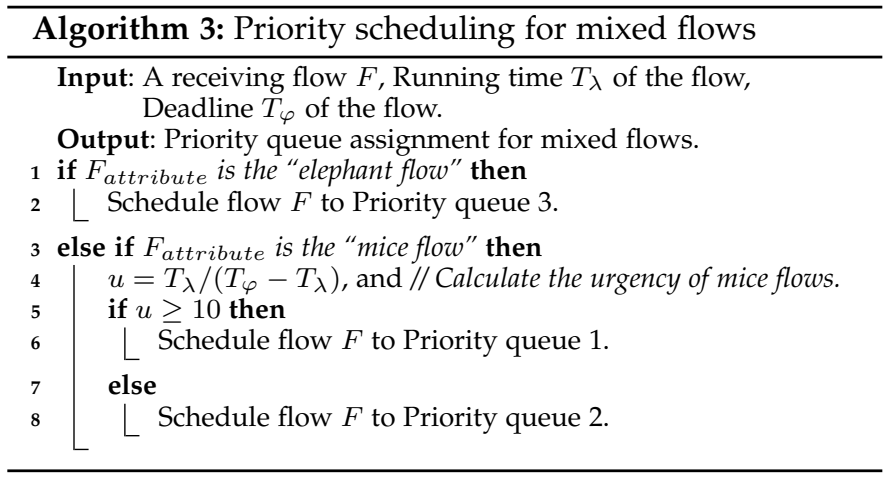

Algorithm 3 shows the execution process for priority scheduling. When the switches received flow $F$, the algorithm first determined its attribute, $F_{\text {attribute }}$. If $F_{\text {attribute }}$ is the "elephant flow" it was scheduled to the lowest-priority queue, 3 . In addition, if $F_{\text {attribute }}$ was the "mice flow" its 
urgency $u$ was calculated. When the value of $u$ exceeded 10 [44], it was scheduled to the high-priority queue, 1; otherwise, it was scheduled to the low-priority queue, 2.

\subsection{Coding-Based Mice Flow Congestion Control}

The existing congestion control schemes DCTCP [9] and $\mathrm{D}^{2} \mathrm{TCP}[10]$ can adaptively adjust the congestion window size of flows according to the network congestion state to reduce their FCT. However, when the switch queue is heavily congested, packet loss can occur, which causes a long retransmission latency.

The retransmission latency caused by packet loss is shown in Fig. 4(a). When a packet is lost, the sender relies on three repeated ACKs to sense packet loss and then resends the packet. The latency caused by loss packet retransmission is two RTTs. To reduce the retransmission latency, we proposed a coding-based transmission protocol to encode the flow through FEC technology [45]. As shown in Fig. $4(\mathrm{~b})$, the sender generated a redundant packet based on five packets by encoding the flow. When a packet is lost, the receiver could receive four packets and a redundant packet, restoring the lost packet by decoding the redundant packet. Thus, the latency caused by loss packet retransmission could be reduced to one RTT through redundant encoding.

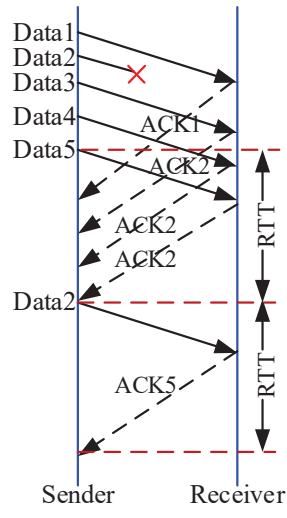

(a)

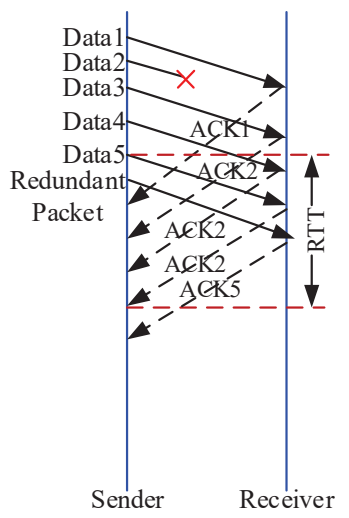

(b)
Fig. 4. The latency comparison: (a) loss packet retransmission, (b) redundancy encoding.

Further, we proposed a coding-based mice flow congestion control to encode the mice flows through the FEC technique [45]. The sender generated redundant packets by encoding the mice flow. When packet loss occurred, the receiver could restore the lost packets by decoding the redundant packets, which reduced the retransmission delay caused by packet loss. In addition, when mice flows were congested, it could adaptively adjust the congestion window of mice flows to reduce their FCT according to the extent of network congestion, $\alpha$, and the urgency, $u$, of mice flows.

On the end-hosts, each $w$ source packet $\left(S_{1}, S_{2}, \ldots, S_{w}\right)$ formed a coding unit, which was encoded into $w+r$ encoded packets $\left(R_{1}, R_{2}, \ldots, R_{w+r}\right)$ using the FEC technique. For each coding unit, the number of redundant packets, $r$, can be changed according to the extent of network congestion, $\alpha$. We used the same ECN marking mechanism as DCTCP [9] to determine the extent of network congestion. When the queue length of the switch cache exceeded the threshold $\delta$, the packet's congestion experienced (CE) bit was marked as 1; otherwise, it was not marked. Then, the receiver copied the CE bit information of the packet to the returned ACK and fed it back to the sender. Specifically, the extent of network congestion $\alpha$ is given by Equation (4).

$$
\alpha=(1-v) \times \alpha+v \times \phi
$$

where $\phi$ denotes the fraction of CE marked packets in the recent window of data, and $v$ is the given weight between the new and old samples.

In HPSTOS, $w$ was set to the congestion window of the mice flows. Based on Equations (3) and (4), we constructed a gamma-correction penalty function similar to $\mathrm{D}^{2} \mathrm{TCP}[10]$, as shown in Equation (5).

$$
\xi=\alpha^{u}
$$

The penalty function $\xi$ was always between 0 and 1 , as $\alpha$ was between 0 and 1 . The congestion window, $w$, of the mice flows was adjusted according to Equation (6).

$$
w= \begin{cases}w \times(1-\xi / 2), & \xi>0 \\ w+1, & \xi=0\end{cases}
$$

If there was no congestion in the network (i.e., $\alpha=0$ ), the penalty function $\xi$, would be 0 , and the window size only grew by one segment. When all on-the-fly packets are CEmarked (i.e., $\alpha=1$ ) then $\xi$ would be 1 , and the window size halved, which is identical to TCP.

We used active queue management to maintain a small queue length, which reduced the queuing delay of mice flows. The queue type of the switch was set to drop tail packets. When network congestion, $\alpha$, exceeded a threshold, $\eta$, packets would be dropped from the tail of the queue [9]. Therefore, as the extent of congestion increased, the packet loss ratio increased. To reduce the retransmission latency caused by packet loss, it was necessary to provide more redundant packets. When packet loss occurred, the receiver recovered the lost packets by decoding redundant packets to reduce the retransmission latency. If no packet loss occurred in the queue, the receiver directly discarded the redundant packets in the encoding unit.

To decode quickly on the receiver, the sender needed to generate more redundant packets, which resulted in a large traffic overhead. Conversely, if only a few redundant packets were generated, the decoding speed was slow [46]. Therefore, redundancy was optimized to achieve a better network performance. To ensure that the receiver could receive at least $w^{\prime}$ encoded packets to achieve lost packet recovery, one constraint is shown in Equation (7), where $w$ is the number of source packets, and $w^{\prime}$ is the number of packets received by the receiver.

$$
(1-\alpha) \times\left(w^{\prime}+r\right) \geq w
$$

To reduce the overhead caused by encoding traffic, the number of redundant packets, $r$, generated by encoding each source packet, is shown in Equation (8).

$$
r=\frac{\alpha}{1-\alpha} \times w=\frac{\alpha}{1-\alpha} \times(1-\alpha / 2) \times w=\frac{2 \alpha-\alpha^{2}}{2-2 \alpha} \times w
$$


For $w+r$ encoded packets, we assumed that there were $n$ lost packets at the receiver. If the receiver successfully decoded the source packets, the number of lost packets should be less than the number of redundant packets, $r$. Therefore, the probability of successful decoding at the receiver is given by Equation (9).

$$
\psi=\sum_{n=0, n \leq r} C_{w+r}^{n} \times \alpha^{n} \times(1-\alpha)^{w+r-n}
$$

In the coding process, our HPSTOS scheme only encoded the mice flows, which accounted for approximately $15 \%$ of the total traffic. Therefore, the HPSTOS had less traffic overhead. Assuming that the ratio of the total amount of elephant flows to mice flows is $y$, the traffic overhead $\gamma$ is as shown in Equation (10).

$$
\gamma=\frac{\alpha}{1+y \times(1-\alpha)}
$$

For the coding-based congestion control algorithm, in Algorithm 4, we elaborate on the specific implementation process of congestion control. First, the queue length of the cache queue was detected, and if its value $Q_{\text {length }}$ exceeded the threshold $\delta$, the subsequent incoming packets were marked by the ECN, which was used to calculate the congestion degree of the queue. Then, the congestion degree $\alpha$ was calculated. If $\alpha$ was greater than $\eta$, the queue was heavily congested, and packet loss could occur. At this time, it was necessary to calculate the number of redundant packets, $r$, added to the mice flows and performed decoding operations on the redundant packets at the receiving end to recover the lost packets. In addition, according to the congestion degree $\alpha$, the congestion window, $w$, of the traffic was adjusted at the source host. Finally, if the congestion degree $\alpha$ did not exceed $\eta$, congestion was manageable and packet loss could be avoided; therefore, only the congestion window of the traffic was adjusted. Accordingly, through code-based congestion control, the FCT of the mice flows could be reduced, and when congestion was severe, the retransmission delay caused by packet loss could also be avoided.

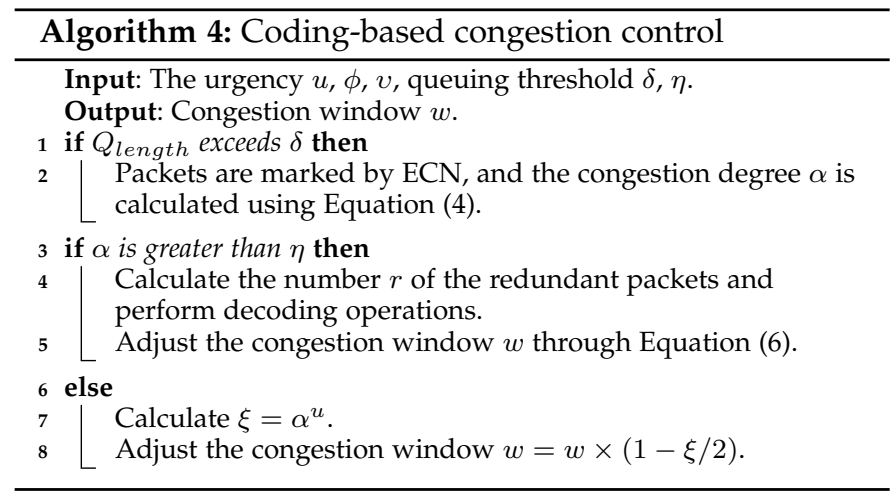

\subsection{Cost-Aware Dynamic Elephant Flow Scheduling}

To improve the throughput of elephant flows, combined with the centralized control of SDN, a cost-aware dynamic elephant flow scheduling algorithm is proposed. To collect flow information, the SDN controller monitored all ports of the switch by periodically sending the port_statistics message. For the collection of link information, the SDN controller sent link layer discovery protocol (LLDP) packets to all switches through the packet_out message. After receiving the LLDP packets, the switch sent the link information to the controller through the packet_in message. Although Hedera [11] and Freeway [12], a controller-based elephant flow scheduling scheme has been proposed, it usually only perceives link utilization and ignores the marginal cost of the link, which reduces the overall network throughput.

In HPSTOS, we can model the network topology using a graph $G(V, E)$, where $V$ is the set of switch nodes and $E$ is the set of links. A set of flows $F$ can be generated between the source and destination servers, and the i-th flow is designated as $f_{i} \in F$. The flow $f_{i}$ is a triple $\left(s_{f_{i}}, d_{f_{i}}, a_{f_{i}}\right)$, where $s_{f_{i}} \in V$ is the set of source switches, $d_{f_{i}} \in V$ is the set of destination switches, and $a_{f_{i}}$ is its size. $P_{f_{i}}$ is the set of all valid paths between $s_{f_{i}}$ and $d_{f_{i}}$ and each flow $f_{i}$ may only select one path from $P_{f_{i}}$ for transmission. We used $N_{k}^{f_{i}}(t)$ as the number of flows $f_{i}$ routed on path $k \in P_{f_{i}}$ at time $t$. The network state $N(t)$ was defined as the number of flows on different paths, as shown in Equation (11).

$$
N(t)=\left(N_{k}^{f_{i}}(t) ; k \in P_{f_{i}}, f_{i} \in F\right)
$$

$T_{l}(t)$ represents the total amount of traffic transmitted on link $l \in E$, as shown in Equation (12).

$$
T_{l}(t)=\sum_{f_{i} \in F} \sum_{k \in P_{f_{i}}, l \in k} a_{f_{i}} N_{k}^{f_{i}}(t)
$$

For each link $l$ with capacity $C_{l}$, when the total amoun$\mathrm{t}$ of traffic is $T_{l}$, we consider a strictly convex function $h\left(T_{l} / C_{l}\right)$, as shown in Equation (13).

$$
h(x)=x^{1+\beta} / 1+\beta,
$$

Here, $\beta$ is a constraint that is greater than 0 . As $x$ increases, $h(x)$ increases and becomes convex. Thus, for every path $k \in P_{f_{i}}$, the function $Y(N(t))$ is given by Equation (14).

$$
Y(N(t))=\sum_{l \in k} h\left(T_{l}(t) / C_{l}\right)
$$

Definition 1 (Link marginal cost). For each link $l$ and flow $f_{i}$, the link marginal cost is defined in following forms.

1) Integral form:

$$
\theta_{l}^{\left(f_{i}\right)}(N(t))=h\left(\frac{T_{l}(t)+a_{f_{i}}}{C_{l}}\right)-h\left(\frac{T_{l}(t)}{C_{l}}\right)
$$

2) Differential form:

$$
\varphi_{l}^{\left(f_{i}\right)}(N(t))=\frac{a_{f_{i}}}{C_{i}} h^{\prime}\left(\frac{T_{l}(t)}{C_{l}}\right)
$$

By defining the link marginal cost [47], we can define the path marginal cost that expresses the increase in the network cost if a flow $f_{i}$ is routed to path $k \in P_{f_{i}}$ at time $t$. The path cost $Z_{k}^{\left(f_{i}\right)}(N(t))$ is given by Equation (17). $Y(N(t))$ represents the network cost before the flow $f_{i}$ is routed to 
path $k$, and $Y(N(t+\Delta t))$ represents the network cost after the flow $f_{i}$ is routed to path $k$.

$$
\begin{aligned}
Z_{k}^{\left(f_{i}\right)}(N(t)) & =Y(N(t+\Delta t))-Y(N(t)) \\
& =\sum_{l \in k}\left[h\left(\frac{T_{l}(t)+a_{f_{i}}}{C_{l}}\right)-h\left(\frac{T_{l}(t)}{C_{l}}\right)\right] \\
& =\sum_{l \in k} \theta_{l}^{\left(f_{i}\right)}(N(t))
\end{aligned}
$$

Similarly, the differential form of the path marginal cost $Z_{k}^{\left(f_{i}\right)}(N(t))$ is shown in Equation (18).

$$
\begin{aligned}
Z_{k}^{\left(f_{i}\right)}(N(t)) & =\frac{\sigma Y(N(t))}{\sigma N_{k}^{f_{i}}(t)} \\
& =\sum_{l \in k} \frac{a_{f_{i}}}{C_{i}} h^{\prime}\left(\frac{T_{l}(t)}{C_{l}}\right) \\
& =\sum_{l \in k} \varphi_{l}^{\left(f_{i}\right)}(N(t))
\end{aligned}
$$

In DCN, our goal is to schedule elephant flows to path $m\left(m \in P_{f_{i}}\right)$ with the least path marginal cost. Path $m$ can be expressed using Equation (19):

$$
m=\arg \min _{k \in P_{f_{i}}} Z_{k}^{\left(f_{i}\right)}(N(t))
$$

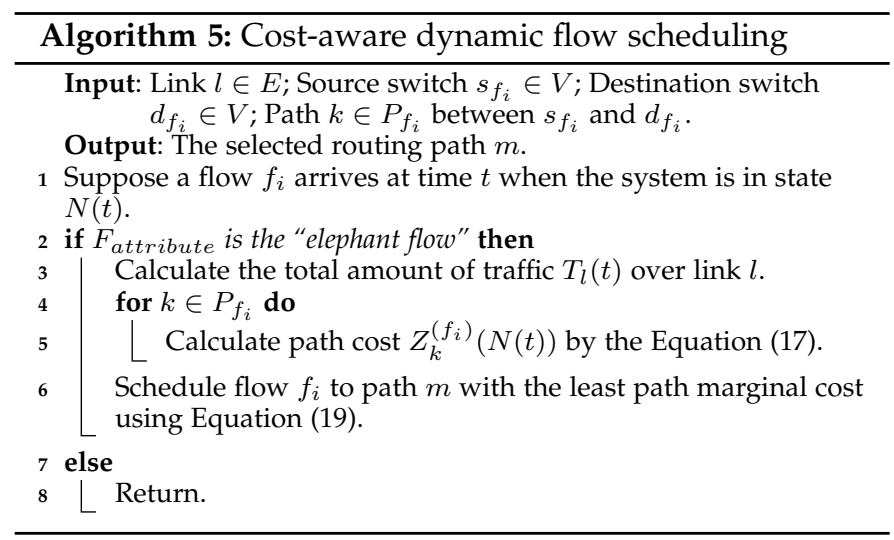

For the cost-aware dynamic flow scheduling algorithm, we display the details of the scheduling procedure in Algorithm 5. First, the algorithm traversed each path between the source and destination servers to calculate the path marginal cost. Then, the marginal cost information of all paths were collected by the controller to form a path status table. Finally, path $m$ with the least path marginal cost was selected through sorting and searching, and the routing table was established on the switch. As each elephant flow arrived, the algorithm was only executed when the routing table did not have a flow entry corresponding to the incoming elephant flow. For the fat-tree topology with $g$ pods, the algorithm could handle $g^{2} / 4$ paths between the source and destination servers. The time complexity of the algorithm was $O\left(g^{2} / 4\right)$.

\subsection{Analysis and Discussion of Performance Con- straints Among Components}

To analyze the relationships and constraints among HPSTOS system components, we adjusted the queuing threshold, $\delta$, set on the priority queue. On the priority scheduling function module, when the queuing threshold was increased, the congestion on the cache queue would increase and the cache queue would become idle. Many packets would be forwarded to the queue, and the queue would be severely congested. Heavy congestion increased the packet loss rate. The proposed coding-based mice flow congestion control encoded the mice flow and recovered the lost packets by decoding redundant packets, which reduced the retransmission delay caused by packet loss and was highly efficiency.

Additionally, when the queuing threshold of the priority queue on the priority scheduling function module was increased, the end-host increased the sending rate of traffic. The sudden increase in traffic increased the load of the elephant flow, and the "hunger" phenomenon appeared on the low priority queue, affecting the overall network throughput. The proposed cost-aware dynamic elephant flow scheduling scheduled the elephant flow to the lightest-load path by minimizing the path marginal cost, which improved the overall throughput of the network and had higher efficiency.

Therefore, through the above analysis, we can see that the various components of the proposed HPSTOS system are closely connected and mutually constrained. Further, as the queuing threshold increased, the performance and efficiency of the designed components were higher than the existing solutions. We have also proved this view through relevant experimental tests in the performance evaluation in Section 5.7.

\section{Performance Evaluation}

In this section, we discuss the evaluation of the performance of the HPSTOS scheme, which was done using the Mininet simulation tool and Ryu controller to build a test platform.

\subsection{Simulation Environment and Parameter Settings}

The Mininet simulation tool is considered reliable. In application, the function code of Mininet can be directly transferred to the physical switch without any modification. Moreover, the HPSTOS scheme was implemented on Ryu software. Ryu is an independent application layer software, which can be directly applied in actual scenarios. The experiment was run on a Dell Inspiron 3647 with a 4-core Intel i5-4460S $2.9 \mathrm{GHz}$ CPU and 8 GB memory, a 1 TB hard disk, and a Realtek RTL81868 Gigabit Ethernet NIC. The PC runs Ubuntu 16.04-64 bit system with Linux 4.11.5 kernel. For the network topology, we employed a 6-pod Fat-Tree hosting 54 servers, and there were 9 paths between the source and destination servers. Each pod contained 3 ToR switches and 3 aggregation switches. Each switch is a 6-port 1 Gbps OVS, and the queue length threshold was 20 packets [9], [10]. In our simulation, we sampled flows at a sampling rate of 0.05 , and defined flows with a size greater than 1 MByte as elephant flows as $85 \%$ of flows in the DCN are smaller than 1 MBytes [48]. The link cost parameter $\beta$ was set to 1 [47] because the optimal objective function of the link cost was the strictly convex function $h(x)=x^{1+\beta} / 1+\beta$, only when the quadratic function $x^{2}$, the strictly convex function had the optimal solution, so the cost parameter $\beta$ was 1 . Further, the maximal capacity of controller was $9 \times 10^{6}$ packets/s 
TABLE 1

Key parameters in our simulation.

\begin{tabular}{lc}
\hline Parameter & Value \\
\hline Link speed & 1 Gbps \\
\hline Switch queue type & Drop Tail \\
\hline Switch queue buffer & 250 packets \\
\hline Sampling rate $\varepsilon$ & 0.05 \\
\hline Threshold of suspected elephant flow $\rho$ & 0.8 MByte \\
\hline Threshold of real elephant flow $\sigma$ & 1 MByte \\
\hline Threshold $\delta$ of queue length & 20 packets \\
\hline Threshold $\eta$ of congestion extent & 0.35 \\
\hline Cost constraint parameter $\beta$ & 1 \\
\hline
\end{tabular}

TABLE 2

Traffic distributions used for evaluation.

\begin{tabular}{lc}
\hline Cumulative Probability & Flow sizes \\
\hline 0 & 0 \\
\hline 0.15 & $10^{4}$ Bytes \\
\hline 0.2 & $2 \times 10^{4}$ Bytes \\
\hline 0.3 & $3 \times 10^{4}$ Bytes \\
\hline 0.4 & $5 \times 10^{4}$ Bytes \\
\hline 0.53 & $8 \times 10^{4}$ Bytes \\
\hline 0.6 & $2 \times 10^{5}$ Bytes \\
\hline 0.7 & $10^{6}$ Bytes \\
\hline 0.8 & $2 \times 10^{6}$ Bytes \\
\hline 0.9 & $5 \times 10^{6}$ Bytes \\
\hline 0.97 & $10^{7}$ Bytes \\
\hline 1 & $3 \times 10^{7}$ Bytes \\
\hline
\end{tabular}

based on OpenFlow 1.2 protocol. The rest of parameters can be found in Table 1.

Additionally, we used actual web search workload [9] data to generate traffic for our experiment. This workload was obtained from production data centers. Table 2 shows the cumulative distribution of flow sizes seen in this workload, and the size of the flow can be randomly obtained by the cumulative distribution function (CDF). This workload was heavy tailed: most flows were small, while a small number of large flows contributed to a substantial portion of the traffic. The time interval achieved by the flow obeyed the Poisson distribution. Flow deadlines were randomly generated using an exponential distribution, with a mean of $20 \mathrm{~ms}$ [9].

\subsection{Comparison Schemes and Evaluation Metrics}

We evaluated the performance of the HPSTOS scheme and compared it with ECMP [49], DCTCP [9], D ${ }^{2}$ TCP [10], Freeway [12] and RepFlow [23]. ECMP forwards packets among equal-cost egress ports using static hashing. DCTCP and $\mathrm{D}^{2} \mathrm{TCP}$ adaptively adjust the flow rate according to the congestion level. Freeway senses the network status through the controller and adaptively schedule traffic to the lighter-load path. RepFlow replicates mice flows to different forwarding paths. In this experiment, the following main evaluation metrics were considered: the overhead of elephant flow detection, accuracy of elephant flow detection, network throughput, flow completion time (FCT), coding delay, and decoding delay. The overhead of elephant flow detection was measured by the number of flow request messages collected by the controller. The accuracy of elephant flow detection was the ratio of the detected size of the elephant flow to its actual size. The network throughput referred to the number of bytes received per unit of time. The FCT was the difference between the time when the last packet of a flow was received by the destination server and the time when the first packet of the same flow was sent from the source server. The other metrics are described in Table 3.

\subsection{Performance Evaluation of Elephant Flow Detec- tion}

For the proposed elephant flow detection method, we evaluated its performance based on detection cost and detection accuracy. As shown in Fig. 5(a), we verified the detection overhead performance by measuring the flow request messages collected by the controller. Compared with the controller-based detection methods Hedera [11] and Helios [13], the detection cost of HPSTOS was much smaller. Because HPSTOS sampled the traffic, it avoided the need for the controller to collect a large number of flow request messages. Under different traffic loads, we could see that the detection overhead of HPSTOS was reduced by at least about $50 \%$ on average compared to Hedera and Helios.

For the detection accuracy of the elephant flow, we evaluated its performance, as shown in Fig. 5(b). As the traffic load increased, the detection accuracy of HPSTOS was better than the sampling-based detection methods NetFlow [18] and sFlow [19]. When the traffic load was 0.6, the detection accuracy of HPSTOS was superior by at least about $50 \%$. Because HPSTOS added flow table identification on the basis of sampling detection, this improved the accuracy of the elephant flow detection.

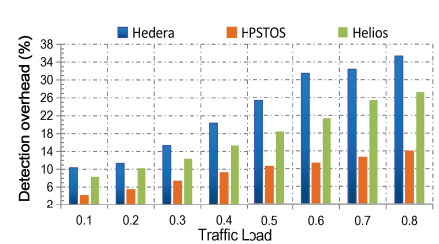

(a)

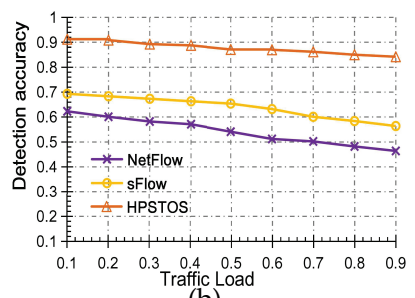

(b)
Fig. 5. (a) Overhead of elephant flow detection; (b) Accuracy of elephant flow detection.

\subsection{Performance Evaluation of FCT, Throughput, and Other Performance Metrics}

As shown in Fig. 6(a), HPSTOS reduced the overall FCT by $\sim 50 \%-55 \%, \sim 40 \%-50 \%, \sim 40 \%$, and $\sim 30 \%$ for loads from 0.5 to 0.9 compared to ECMP, DCTCP, $\mathrm{D}^{2} \mathrm{TCP}$, and RepFlow, respectively. Fig. 6(b) shows that HPSTOS reduced the mean FCT of mice flows by $\sim 50 \%$ and $\sim 40 \%$ for loads from 0.3 to 0.8 compared to ECMP and DCTCP, respectively. This is due to HPSTOS preferentially transmitting the mice flows and using redundant coding to reduce the delay of the mice flows. The drawback of priority transmission was the longer FCT of elephant flows, shown in Fig. 6(c). HPSTOS increased the mean FCT of elephant flows by $\sim 30 \%$ compared to Freeway. As shown in Fig. 6(d), HPSTOS had a higher packet loss ratio than $\mathrm{D}^{2} \mathrm{TCP}$. This could be attributed to an increase in the number of redundant packets, thereby increasing the packet loss rate of HPSTOS as redundant coding can result in longer queue lengths. 
TABLE 3

Evaluation metrics used in our experiments

\begin{tabular}{ll}
\hline Metrics & Descriptions \\
\hline Overhead of elephant flow detection & The overhead of flow request messages processed by the controller. \\
\hline Accuracy of elephant flow detection & the ratio of the detection size of the elephant flow to its actual size. \\
\hline Network throughput & The number of bytes received per unit of the time. \\
\hline Flow completion time & The transmission time of the flow from the source to the destination. \\
\hline Coding delay & The average transmission time of encoded packets. \\
\hline Decoding delay & The decoding time of redundant packets. \\
\hline Flow request message & The Packet_in message sent by the switch to the controller. \\
\hline
\end{tabular}

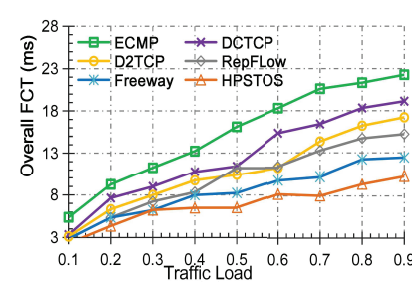

(a)

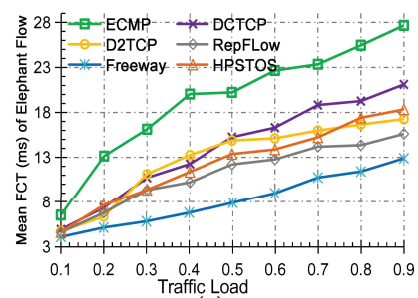

(c)

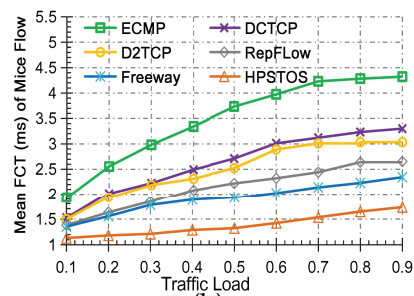

(b)

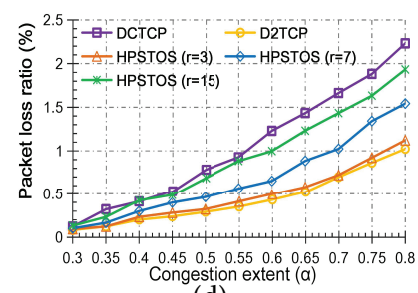

(d)
Fig. 6. The performance comparison: (a) overall FCT; (b) mean FCT of mice flows; (c) mean FCT of elephant flows; (d) packet loss ratio.

As shown in Fig. 7(a) and (b), HPSTOS performed better than other strategies for both the overall throughput and elephant flow throughput. Overall throughput were increased by $\sim 35 \%-60 \%, \sim 30 \%-40 \%, \sim 30 \%$ and $\sim 20 \%$ for loads from 0.3 to 0.9 compared to ECMP, DCTCP, $\mathrm{D}^{2} \mathrm{TCP}$, and RepFlow, respectively. Elephant flow throughput were increased by $\sim 60 \%, \sim 30 \%-50 \%, \sim 35 \%$ and $\sim 20 \%$ for loads from 0.2 to 0.8 compared to ECMP, DCTCP, RepFlow, and Freeway, respectively. For load balancing, HPSTOS increased average link utilization by $\sim 50 \%, \sim 40 \%-55 \%$ and $\sim 30 \%-50 \%$ for loads from 0.1 to 0.9 when compared to ECMP, DCTCP, and RepFlow, respectively, as shown in Fig. 7(c). These results are due to HPSTOS scheduling elephant flows to light load paths by constraining the link marginal cost.

As shown in Fig. 8, we tested the average transport latency between hosts under different schemes. As the traffic load increased, the average transport latency increased due to traffic bursts that increase the congestion of the link. HPSTOS reduced the average latency by $\sim 33 \%-41 \%$ and $\sim 19 \%-27 \%$ for loads from 0.1 to 0.9 over ECMP, and DCTCP, respectively. As ECMP did not make any differentiation between elephant and mice flows, the mice flows could be blocked in the congested nodes and needed to compete for bandwidth with elephant flows. Additionally, under different traffic loads, the average latency of HPSTOS was similar to $\mathrm{D}^{2} \mathrm{TCP}$, RepFlow, and Freeway.

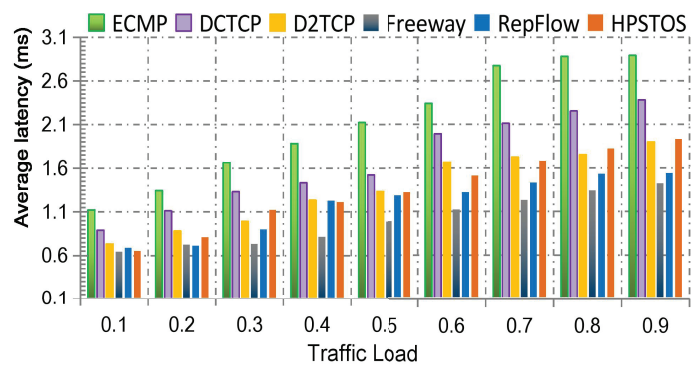

Fig. 8. Average transport latency.

\subsection{Advantages of Encoding Mice Flows}

To show the advantages of using coding to reduce delays, we compared the delay performance of schemes with and without coding. The delay is the average transmission time of the packet, and it refers the time between when the packet enters the network and when it leaves. Fig. 9(a) shows, as the extent of traffic congestion, $\alpha$, increased, HPSTOS with coding performed better than DCTCP and $\mathrm{D}^{2} \mathrm{TCP}$ without coding in delay performance. HPSTOS reduced the retransmission delay caused by packet loss using coding. Specifically, when $\alpha$ was 0.35 , the delay of HPSTOS was reduced by $45 \%$ compared with DCTCP. When the number of redundant packets is small, such as $r=3$, decoding fails and packets are re-transmitted, resulting in a large delay.

As shown in Fig. 9(b), we analyzed the decoding delay of HPSTOS under different numbers of redundant packets. As the number of redundant packets increased, the decoding delay of HPSTOS decreased as more redundant packets results in faster the decoding. In Fig. 9(c) it is shown that packet coding reduced transmission delay by at least $20 \%$ compared to packet replication. The traffic overhead of HPSTOS is shown in Fig. 9(d), $y$ is the ratio of total amount of elephant flows to mice flows. As $\alpha$ increased, the traffic overhead was less than $8 \%$. Therefore, the traffic overhead of HPSTOS is relatively small due to the HPSTOS scheme only encoding the mice flows, which account for about $15 \%$ of the total traffic.

\subsection{Performance Evaluation of Scalability Metrics}

As shown in Fig. 10(a), we evaluated the controller's overhead for processing flow request messages. The flow request message was generated when a flow arrived at the switch and no flow table entry matched it. The switch would send a packet_in message to the controller to calculate the forwarding rule. HPSTOS reduced the request message by $\sim 50 \%$ for loads from 0.3 to 0.8 over Freeway because 


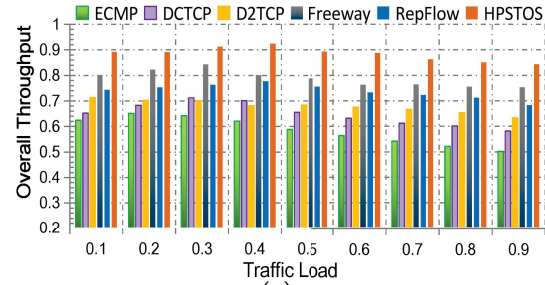

(a)

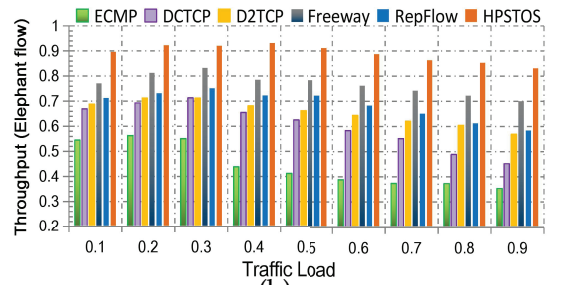

(b)

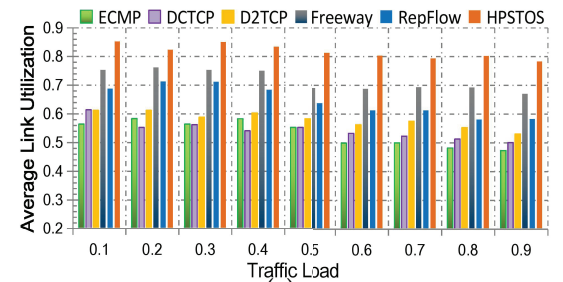

(c)

Fig. 7. The performance comparison: (a) overall throughput; (b) throughput of elephant flows; (c) average link utilization.

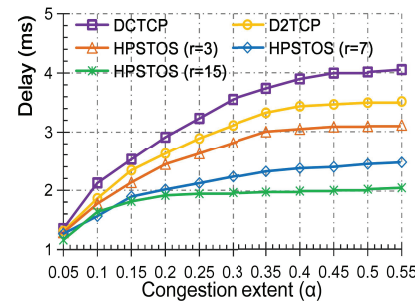

(a)

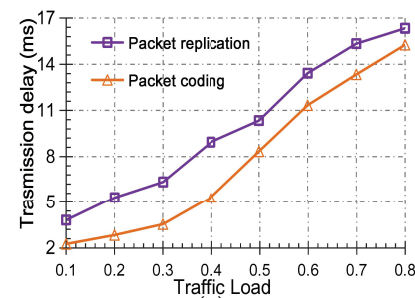

(c)

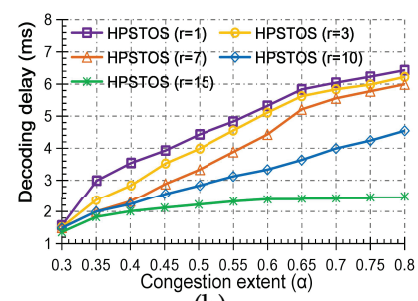

(b)

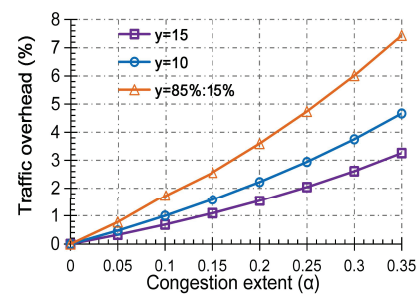

(d)
Fig. 9. The performance comparison: (a) overall delay; (b) decoding delay; (c) transmission delay; (d) Traffic overhead.

HPSTOS only handles request messages of elephant flows. In Fig. 10(b), the end-host's overhead in processing ECN messages is evaluated. HPSTOS reduced ECN messages by $\sim 30 \%$ compared to DCTCP and $\mathrm{D}^{2} \mathrm{TCP}$ under different loads. Therefore, compared with current schemes, HPSTOS has better scalability.

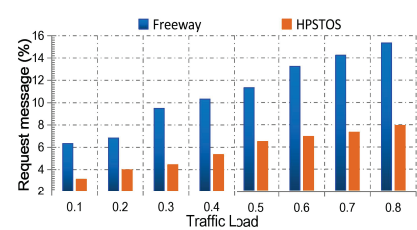

(a)

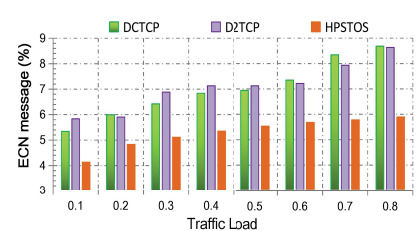

(b)
Fig. 10. (a) flow request messages; (b) ECN messages.

\subsection{Impact of Queuing Threshold}

In order to verify the performance constraints among components of the HPSTOS system, we performed performance evaluation of the FCT of the mice flow and the throughput of the elephant flow under different queuing thresholds. As shown in Fig. 11(a), as the queuing threshold increased, the FCT of mice flows showed an increasing trend. This is due to the fact that increasing the queuing threshold will increase the blocking rate of the queue. Under different queuing thresholds, compared with the congestion control schemes DCTCP and $\mathrm{D}^{2} \mathrm{TCP}$, the FCT of HPSTOS was reduced by about $35 \%$ on average. Because HPSTOS encoded the mice flow, retransmission delay caused by packet loss could be reduced.

As shown in Fig. 11(b), under different queuing thresholds, the throughput of HPSTOS was better than controllerbased flow scheduling schemes Hedera and Freeway. Compared to Hedera and Freeway, HPSTOS's throughput was reduced by an average of about $30 \%$ and $20 \%$, respectively. Because HPSTOS could perceive the marginal cost of the link, it scheduled the elephant flow to the path with the lightest load when congestion was heavy.

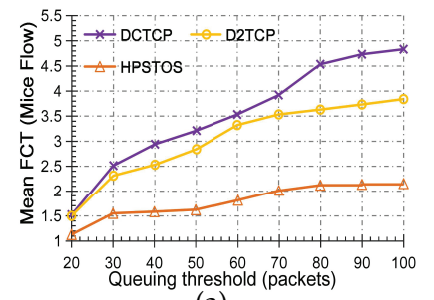

(a)

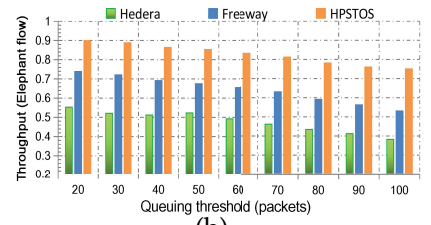

(b)
Fig. 11. (a) FCT of mice flows; (b) Throughput of elephant flows.

\section{Conclusion}

In this paper, we propose a high-performance and scalable traffic optimization strategy (HPSTOS) for mixed flows. In order to distinguish between elephant flows and mice flows, HPSTOS detects elephant flows through flow sampling and flow table recognition. HPSTOS jointly uses distributed and centralized mechanisms to achieve higher network performance and scalability. On the end-host, HPSTOS adaptively adjusts the sending rate of the mice flow through codingbased congestion control. Further, on the switch, HPSTOS schedules the mice flow to the high priority queue through priority scheduling. On the centralized controller, HPSTOS dynamically schedules the elephant flow to the path with lighter load through cost-aware flow scheduling. Performance evaluation shows that HPSTOS has better network performance and scalability when compared with current strategies. For future work, we will focus on the placement of multi-controllers to support further scalability of HPSTOS in large-scale networks.

\section{ACKNOWLEDGMENTS}

This work was supported in part by the National Key R\&D Program of China under Grant 2018YFE0202800, the National Natural Science Foundation of China under Grant 
61634004 and 61934002, the Natural Science Foundation of Shaanxi Province for Distinguished Young Scholars under Grant No. 2020JC-26, the Fundamental Research Funds for the Central Universities under Grant No. JB190105, the Open Project Program of the State Key Laboratory of Mathematical Engineering and Advanced Computing under Grant No. 2019A01, and the China Postdoctoral Science Foundation No. 2018M633465.

\section{REFERENCES}

[1] Z. Guo, J. Duan, and Y. Yang, "On-line multicast scheduling with bounded congestion in fat-tree data center networks", IEEE Journal on Selected Areas in Communications, vol. 32, no. 1, pp. 102-115, 2014.

[2] Z. Guo and Y. Yang, "Exploring server redundancy in nonblocking multicast data center networks", IEEE Transactions on Computers, vol. 64, no. 7, pp. 1912-1926, 2015.

[3] K. Zhen, X. Wang, and J. Liu, "Distributed Traffic Flow Consolidation for Power Efficiency of Large-scale Data Center Network", IEEE Transactions on Cloud Computing, pp. 1-1, 2020.

[4] K. Zhen, Y. Bai, and X. Wang, "FCTcon: Dynamic Control of Flow Completion Time in Data Center Networks for Power Efficiency", IEEE Transactions on Cloud Computing, pp. 1-1, 2019.

[5] F. Tang, H. Zhang, L.T. Yang, and L. Chen, "Elephant Flow Detection and Differentiated Scheduling with Efficient Sampling and Classification", IEEE Transactions on Cloud Computing, pp. 1-1, 2019.

[6] L. Chen, J. Lingys, K. Chen, and F. Liu, "Auto: Scaling deep reinforcement learning for datacenter-scale automatic traffic optimization", ACM Special Interest Group on Data Communication, pp. 191-205, 2018.

[7] S. Kandula, S. Sengupta, A. Greenberg, P. Patel, and R. Chaiken, "The nature of data center traffic: measurements and analysis", Proc. 9th ACM SIGCOMM conference on Internet measurement conference, pp. 202-208, 2009.

[8] W. Wang, Y. Sun, K. Salamatian, and Z. Li, “Adaptive Path Isolation for Elephant and Mice Flows by Exploiting Path Diversity in Datacenters", IEEE Transactions on Network and Service Management, vol. 13, no. 1, pp. 5-18, 2016.

[9] M. Alizadeh, A. Greenberg, D. Maltz et al., "Data center TCP (DCTCP)", ACM Special Interest Group on Data Communication (SIGCOMM), vol. 41, no. 4, pp. 63-74, 2011.

[10] B. Vamanan, J. Hasan, T. Vijaykumar et al., "Deadline-aware datacenter TCP (D2TCP)", ACM Special Interest Group on Data Communication (SIGCOMM), vol. 42, no. 4, pp. 115-126, 2012.

[11] M. AI-Fares, S. Radhakrishnan, B. Raghavan et al., "Hedera: Dynamic Flow Scheduling for Data Center Networks", Proc. NSDI, pp. 19-19, 2010.

[12] W. Wang, Y. Sun, K. Zheng et al., "Freeway: Adaptively isolating the elephant and mice flows on different transmission paths", IEEE ICNP, pp. 362-367, 2014.

[13] N. Farrington, G. Porter, S. Radhakrishnan et al., "Helios: a Hybrid Electrical/Optical Switch Architecture for Modular Data Centers", ACM SIGCOMM, vol. 40, no. 4, pp. 339-350, 2010.

[14] A. AlGhadhban, B. Shihada, "FLight: A Fast and Lightweight Elephant-Flow Detection Mechanism", Proc. of IEEE 38th International Conference on Distributed Computing Systems (ICDCS 2018), pp. 1537-1538, 2018.

[15] A. Tavakoli, M. Casado, T. Koponen, and S. Shenker, "Applying NOX to the Data center", Proceedings of workshop on Hot Topics in Networks (HotNets-VIII), pp. 1-6, 2019.

[16] A.R. Curtis, W. Kim, and P. Yalagandula, "Mahout: Low-overhead datacenter traffic management using end-host-based elephant detection", Proc. IEEE INFOCOM, pp. 1629-1637, 2011.

[17] A.R. Curtis, J.C. Mogul, J. Tourrilhes et al., "DevoFlow: scaling flow management for high-performance networks", ACM SIGCOMM, vol. 41, no. 4, pp. 254-265, 2011.

[18] The internet society. Cisco systems NetFlow services export version 9 [EB/OL]. (2004-10) [2019-04-15]. http://www. rfceditor.org/rfc/rfc3954.

[19] InMon. Traffic Monitoring using sFlow [EB/OL]. (2013-03) [201904-15]. https://sflow.org/sFlowOverview.pdf.

[20] T. Mori, M. Uchida, R. Kawahara, J. Pan, and S. Goto, "Identifying elephant flows through periodically sampled packets", Proc. the 4th ACM SIGCOMM conference on Internet measurement, pp. 115-120, 2004.
[21] C. Wilson, H. Ballani, T. Karagiannis, and A. Rowtron, "Better never than late: Meeting deadlines in datacenter networks", Proc. ACM SIGCOMM, pp. 50-61, 2011.

[22] C. Hong, M. Caesar, and P.B. Godfrey, "Finishing flows quickly with preemptive scheduling", Proc. ACM SIGCOMM, vol. 42, no. 4, pp. 127-138, 2012.

[23] H. Xu, B. Li, "RepFlow: Minimizing flow completion times with replicated flows in data centers", IEEE INFOCOM, pp. 1581-1589, 2014.

[24] F.J. Corbato, and R.C. Daley, "An Experimental Time-sharing System", Proc. of ACM Spring Joint Computer Conference, pp. 335-344, 1962.

[25] W. Bai, L. Chen, K. Chen, D. Han et al., "Information-Agnostic Flow Scheduling for Commodity Data Centers", Proc. of USENIX NSDI, pp. 455-468, 2015.

[26] L. Chen, K. Chen, W. Bai, and M. Alizadeh, "Scheduling mixflows in commodity datacenters with karuna", Proc. of the ACM SIGCOMM Conference, pp. 174-187, 2016.

[27] M. Alizadeh, S. Yang, M. Sharif et al., "pFabric: Minimal Nearoptimal Datacenter Transport", Proc. of ACM SIGCOMM, vol. 43, no. 4, pp. 435-446, 2013.

[28] Y. Wang, X. Tao, Q. He, Y. Kuang, "A dynamic load balancing method of cloud-center based on SDN", China Communications, vol. 13, no. 2, pp. 130-137, 2016.

[29] J. Perry, A. Ousterhout, H. Balakrishnan et al., "Fastpass: a centralized zero-queue datacenter network", SIGCOMM Comput. Commun, vol. 44, no. 4, pp. 307-318, 2014.

[30] F. Carpio, A. Engelmann, and A. Jukan, “DiffFlow: Differentiating Short and Long Flows for Load Balancing in Data Center Networks", Proc. of USENIX NSDI, pp. 337-350, 2016.

[31] A. Dixit, P. Prakash, Y. Hu, and R.R. Kompella, "On the impact of packet spraying in data center networks", Proc. IEEE INFOCOM, pp. 2130-2138, 2013.

[32] S. Kandula, D. Katabi, S. Sinha, and A. Berger, "Dynamic load balancing without packet reordering", ACM SIGCOMM Computer Communication, pp. 51-62, 2007.

[33] S. Sen, D. Shue, S. Ihm, and M.J. Freedman, "Scalable, optimal flow routing in datacenters via local link balancing", Proc. ACM CoNEXT, pp. 151-162, 2013.

[34] C. Raiciu, S. Barre, C. Pluntke et al., "Improving datacenter performance and robustness with multipath TCP", ACM SIGCOMM Computer Communication, pp. 266-277, 2011.

[35] M. Alizadeh, T. Edsall, S. Dharmapurikar et al., "CONGA: Distributed congestion-aware load balancing for datacenters,", $A C M$ conference on SIGCOMM, pp. 503-514, 2014.

[36] K. He, E. Rozner, K. Agarwal et al., "Presto: Edge-based load balancing for fast datacenter networks,", ACM SIGCOMM Computer Communication, pp. 465-478, 2015.

[37] E. Vanini, R. Pan, M. Alizadeh et al., "Let it flow: Resilient asymmetric load balancing with flowlet switching,", 14th Symposium on Networked Systems Design and Implementation (NSDI), pp. 407-420, 2017.

[38] S. Ghorbani, Z. Yan, P.B. Godfrey, Y. Ganjali, and A. Firoozshahian, "Micro load balancing in data centers with DRILL,", ACM Workshop on Hot Topics in Networks, pp. 1-7, 2015.

[39] H. Zhang, J. Zhang, W. Bai, K. Chen, and M. Chowdhury, "Resilient Datacenter Load Balancing in the Wild,", ACM Special Interest Group on Data Communication, pp. 253-266, 2017.

[40] N. Katta, M. Hira, C. Kim et al., "HULA: Scalable load balancing using programmable data planes,", Proceedings of the Symposium on SDN Research, pp. 1-12, 2016.

[41] X. Wu, and X. Yang, "DARD: Distributed adaptive routing for datacenter networks", IEEE International Conference on Distributed Computing Systems, pp. 32-41, 2012.

[42] C. Xing, K. Ding, C. Hu, and M. Chen, "Sample and fetch-based large flow detection mechanism in software defined networks", IEEE Communications Letters, pp. 1764-1767, 2016.

[43] C. Ding, and R. Rojas, "DAQ: deadline-aware queue scheme for scheduling service flows in data centers", IEEE International Conference on Communications, pp. 2989-2994, 2014.

[44] T. Wang, H. Xu, and F. Liu, "Aemon: information-agnostic mixflow scheduling in data center networks", Asia-Pacific Workshop on Networking, pp. 106-112, 2017.

[45] K. Sundararajan, D. Shah, M. Medard et al., "Network coding meets TCP", IEEE INFOCOM, pp. 280-288, 2009. 
[46] J. Hu, J. Huang, W. Lv et al., "CAPS: Coding-based Adaptive Packet Spraying to Reduce Flow Completion Time in Data Center", IEEE INFOCOM, pp. 2294-2302, 2018.

[47] M. Shafiee and J. Ghaderi, "A Simple Congestion-Aware Algorith$\mathrm{m}$ for Load Balancing in Datacenter Networks", IEEE Transactions on Networking, pp. 3670-3682, 2017.

[48] CAIDA. Anonymized internet traces [EB/OL]. (2014-10) [2019-0417]. http://www.caida.org/data/passive_2014_dataset.xml.

[49] P. Gill, N. Jain, and N. Nagappan, "Understanding Network Failures in Data Centers: Measurement, Analysis, and Implications", Proc. ACM SIGCOMM, pp. 350-361, 2011.

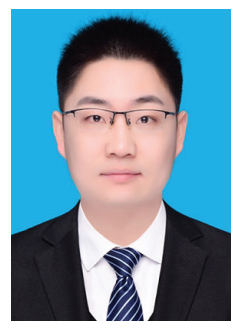

Yong Liu received the M.E. degree in Computer Application Technology from Henan University, Kaifeng, China, in 2016. Currently, he is pursuing the Ph.D degree in Telecommunication and Information Systems in the Stata key lab of ISN, Xidian University, China. His research interests include data center networks, software defined networking, and optical interconnected networks.

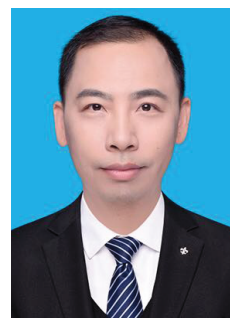

Huaxi Gu is a professor affiliated with the State Key Lab of ISN, Xidian University. Prof. Gu is the leader of the Youth Innovation Team of Shaanxi Universities. He is leading a project as the principal investigator, funded by the National Key Research and Development Program of China. $\mathrm{He}$ is also the principal investigator for one key, two general and one youth project funded by National Natural Science Foundation. Prof. Gu has published over 200 journal and conference papers, with his research interests being in the areas of networking technologies, network on chip, optical interconnect, etc. Prof. Gu served as the TPC member of GLOBECOM, ICC, PDCAT, etc., and the technical reviewer for multiple journals including IEEE Transactions on Computers, IEEE Transactions on VLSI, IEEE Transactions on Cloud Computing, IEEE/OSA Journal of Lightwave Technology, etc.

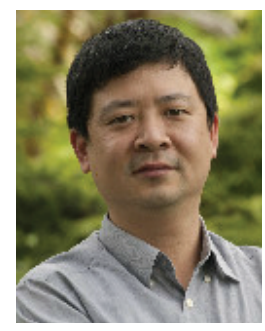

Ning Wang (SM'17) received his Ph.D degree in electronic engineering from the Centre for Communication Systems Research (CCSR), University of Surrey, UK in 2004. He is currently a full professor at the Institute for Communication Systems University of Surrey. Professor Wang's research interests include future network design, $5 \mathrm{G}$ networks, network optimizations and network and service management. 